\title{
Anorectal polyp
}

\author{
R. Bansal ${ }^{1}$, H. Ghanta ${ }^{2}$, R. Blue ${ }^{3}$, R. Sharma ${ }^{1}$
}

(1) Division of Gastroenterology and Hepatology, Elmhurst Hospital Center, New York, USA ; (2) Department of Surgery, Elmhurst Hospital Center, New York, USA ; (3) Department of Radiology, Elmhurst Hospital Center, New York, USA.

A 71-year-old female with hypertension presented with painless rectal bleeding and found to have a soft mass on rectal exam. The colonoscopy revealed a large pedunculated polyp with patchy discoloration arising from the dentate line (Fig. 1). Biopsies of the polyp were obtained.

\section{What is the etiology?}

Biopsies of the polyp were consistent with poorly differentiated amelanotic mucosal melanoma. Immunostains showed that tumor cells were positive for Melan-A, HMB-45 (Fig. 2), S-100 and synaptophysin. The Ki-67 proliferation index was $60 \%$. Molecular testing was negative for BRAF. A staging positron emission tomography-computerized tomography (PETCT) scan showed a metabolically active lesion in the rectum. A thorough skin examination by dermatology did not find any lesions concerning for dysplastic nevi. The patient was offered abdominoperineal resection (APR) but agreed to wide local excision of the polyp. Gross examination revealed a $3.5 \times 2.5 \times 2.0 \mathrm{~cm}$ hemorrhagic, lobulated and friable mass. The microscopic examination showed evidence of vascular invasion. After few weeks of initial diagnosis, patient agreed to APR. She was found to have multiple ulcerated nodules in the liver consistent with metastatic malignant melanoma on excisional biopsy, so APR was aborted. Repeat PET-CT scan showed multiple metabolically active lesions in the liver. The patient started treatment with pembrolizumab and tolerating well.

Mucosal melanomas are a rare subtype of melanoma and account for approximately one percent of all melanomas. The majority of mucosal melanoma arise from the resident melanocytes of mucosal epithelium lining the head and neck, gastrointestinal and genitourinary tract. Mucosal melanomas usually carry a worse prognosis than cutaneous melanomas (1). Primary anorectal melanoma is an uncommon and highly aggressive tumor. Most gastrointestinal melanomas are commonly metastasis from a primary oculocutaneous lesion (2). Surgical excision is the treatment of choice and can range from wide local excision or endoscopic mucosal resection to an APR. The overall prognosis of anorectal melanoma is poor due to their unique biology and tendency for early metastasis regardless of the primary surgical procedure (3).

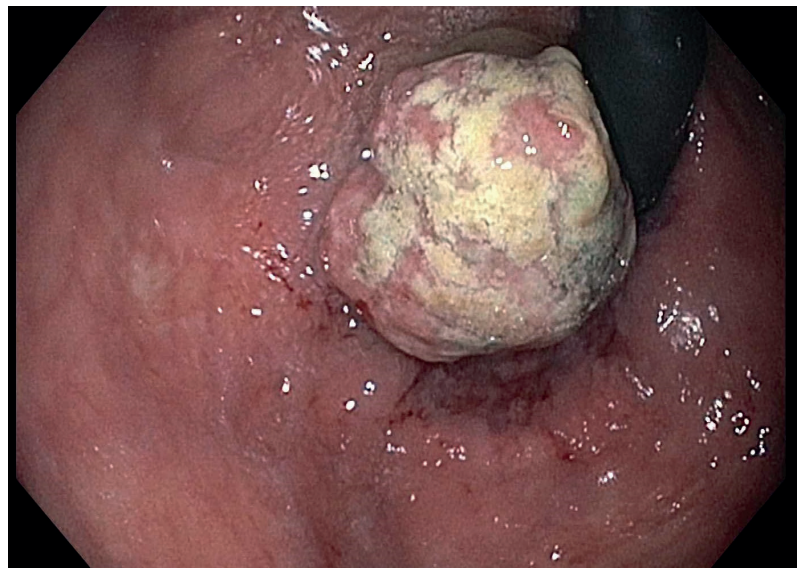

Figure 1.

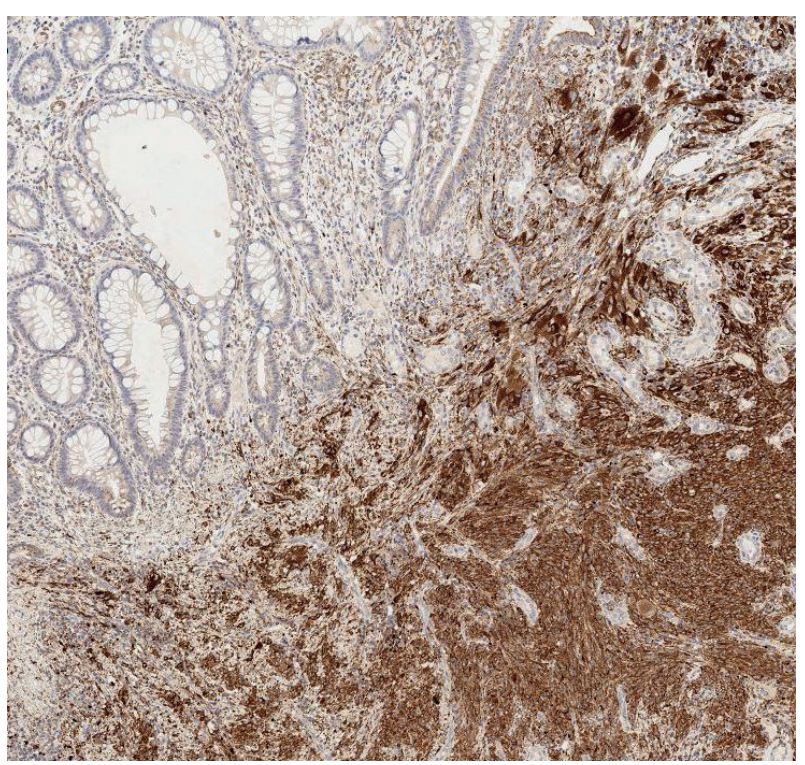

Figure 2.

\section{Conflict of Interest}

All authors declare that no conflicts of interest or financial relationships exist

Correspondence to : Dr. Raghav Bansal, Elmhurst Hospital Center, Assistant Professor, Ichan School of Medicine, Room D3-24D, 79-01 Broadway, Elmhurst, NY 11373, Office 7183342289. Fax 7183341738.

Email : raghav.bansal@mountsinai.org

Submission date : 28/05/2020

Acceptance date : 06/07/2020 


\section{References}

1. KUK D, SHOUSHTARI AN, BARKER CA, PANAGEAS KS, MUNHOZ RR, MOMTAZ P et al. Prognosis of Mucosal, Uveal, Acral, Nonacral Cutaneous, and Unknown Primary Melanoma From the Time of First Metastasis. Oncologist. 2016, 21(7) : 848 .
2. IADEVAIA MD, SGAMBATO D, MIRANDA A, FERRANTE E, FEDERICO A, SASSO FC et al. Amelanotic metastatic melanoma of the stomach presenting with iron deficiency anemia. Acta Gastroenterol. Belg. 2017 AprJun, 80(2) : 327-328.

3. MENON H, PATEL RR, CUSHMAN TR, AMINI A, SEYEDIN SN, ADAMS $\mathrm{AC}$ et al. Management and Outcomes of Primary Anorectal Melanoma in the United States. Future Oncol. 2020 Mar, 16(8) : 329-338. 